\title{
Image Analysis for Calculation of the Toxicity Degree of Cells in Phase Contrast Microscopy Images
}

\author{
M. Athelogou ${ }^{1}$, M. Eblenkamp ${ }^{2}$, G. Schmidt ${ }^{1}$, F. Novotny ${ }^{2}$, E. Wintermantel $^{2}$, \\ G. Binnig ${ }^{1}$ \\ ${ }^{1}$ Definiens AG, Munich \\ ${ }^{2}$ Institute for Medical Engineering, Technical University Munich \\ mathelogou@definiens.com
}

\begin{abstract}
Abstract: Because of the very special type of contrast in phase-contrast images, it is almost impossible to perform fully automated single-cell analysis and quantification successfully. Because fluorescent dyes are highly toxic, phase-contrast images are commonly used to monitor live cells. In this paper, we present a method for the fully automated segmentation, classification and quantification of individual cell morphology in phase-contrast images. We calculate the confluence of the cell population and quantify the degree of toxic damage to each individual cell following phenol incubation. The results are then compared to standard cytotoxicity assays.
\end{abstract}

\section{Introduction}

Phase contrast images are commonly used in order to study cell migration, cell tracking and cell behavior like cell division under different conditions. The morphology of each individual cell correlates with the degree of damage caused by, for example, toxic substances in cell cultures or endogenous toxins. Corresponding calculations of such toxicity damages are usually based on manual or semi-automated methods. Automated methods of differentiated live cell monitoring, based on image analysis algorithms, are limited; they depend upon the degree of confluence of the corresponding cells and on the morphological complexity. Sophisticated segmentation algorithms are applied in order to achieve a robust segmentation of in vitro cell culture images observed with a standard phase-contrast microscope or with video microscopy. Although these algorithms provide a good separation of cell regions from the image background, they are not able to classify cells on a differentiated level, e.g. they do not reliably separate attached cells automatically [1].

\section{Materials and Methods}

To quantify toxicity in fibroblast cell cultures (Fig. 1), a data set of phasecontrast images was acquired. Cell cultivation was based on the ISO-Norm 
10993 protocol. Phenol was used for the induction of cytotoxicity. Images were derived from a Zeissmicroscope, with 10x and 20x objectives, as 8-bit TIFFS. In order to evaluate the quantification of cell damage based on morphological criteria, standard cytotoxicity assays were applied in parallel:

1. LDH assay: The degree of cell damage correlates with the concentration of lactate dehydrogenase $(\mathrm{LDH})$ in the cell culture medium released by damaged cells. The LDH content is quantified by the use of a color reaction induced by LDH.

2. Live/Dead assay: In this differentiating fluorescence based staining Propidiumiodid, which permeates only the membrane of damaged cells is used to stain the nucleus.

For the image analysis we used Definiens XD, which is an application of the Definiens Cognition Network Technology. The Cognition Network Language $(\mathrm{CNL})$ is the corresponding graphical user interface meta-language, which allows efficient development of rule-based algorithms. CNL consists of four basic data structures: processes, domains, image objects and image object classes and supports the use of specific expert knowledge within rule sets. We developed an image analysis solution (CNL rule set), which uses different "maps", where the same phase-contrast image is copied into different instances (maps) for the application of independent different processing procedures. Various algorithms for segmentation and classification were applied to different maps in order to achieve in their combination an optimal segmentation results. Analysis results from one map were used as context for the analysis of objects in other maps; in this way, the same phase contrast image could be analyzed simultaneously differently by concentrating on different aspects and by combining those aspects stepwise into one final result. Context-neutral and context-sensitive features are defined in order to describe the individual properties of cells, the relationships of cells to their neighborhoods and the cell organelles. The degree of confluence
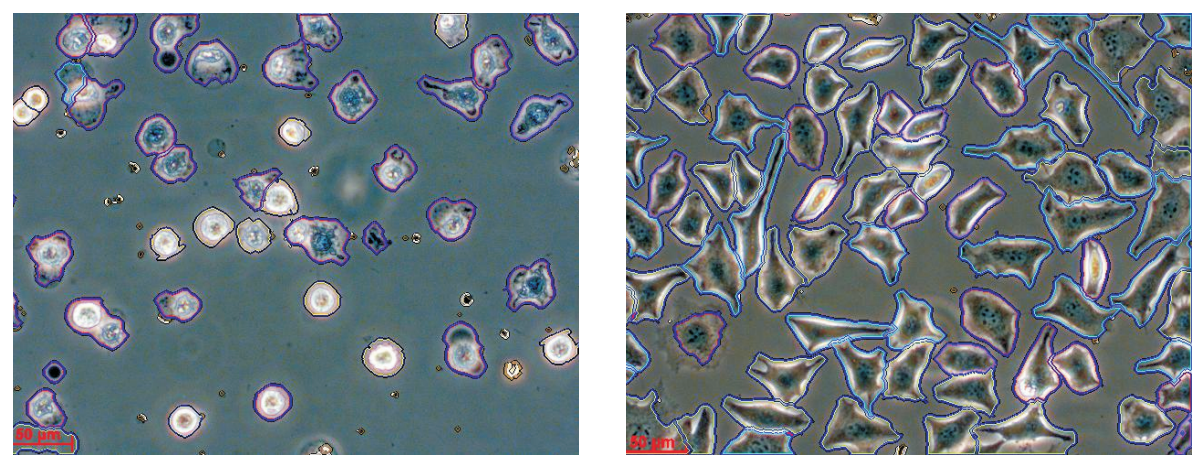

Fig. 1. Single-cell segmentation in phase-contrast images of different confluence using a CNL rule set. Boundaries of the individual cells are highlighted. Left: low confluence and damaged cells; right: high confluence and intact cells. 
of the cell population in an image was calculated as the ratio of the total area of all cells in an image to the sum of the overall area of cell regions plus background. The separation of the corresponding image objects-such as cell regions and image background-requires a precise segmentation of these objects. Therefore, we used multi-resolution segmentation and applied pixel-based filters, such as the edge detection filter, on such individual image objects $[2,3]$. The CNL rule set separates cell clusters into individual cells, segments and extracts individual cell compartments-such as vacuoles and cell protrusions-and classifies the individual cells according to overall morphology, individual sub cellular structures. Cells with a high degree of damage contain several vacuoles and usually lack prominent protrusions. Such features are used for evaluating the individual degree of damage of each individual cell. The total degree of damage was calculated as the average cell damage of all individual cells in an image. To calculate the degree of damage of individual cells, we developed a method that calculates prominent morphological parameters. In terms of the two classes of round cells in the images-mitotic cells and dead cells-mitotic cells are rounder and have smoother boundaries than dead cells. Cells that show halos (surrounding light-colored areas) are usually damaged. Other kinds of cells, flat extended cells in close proximity to the substrate, showing little or no protrusions are also usually damaged (Fig. 1); as well as those with vacuoles in their nuclei. All these parameters were used to define a "Damage Factor Single Cell" (DFSC) for each individual cell

$$
\text { DFSC } \left.\left.\left.\sim f_{1} \text { (protrusions }\right)+f_{2} \text { (vacuoles }\right)+f_{3}(\text { halos })+f_{4} \text { (roundness }\right)
$$

The coefficients $f_{1}, f_{2}, f_{3}, f_{4}$ are calculated automatically using the developed rule set and according to the numerical values of the corresponding morphological parameters of individual cells. The mean value of the DFSC over all individual cells in an image is defined as the "damage factor" for the image. The ratio of this "damage factor" to the confluence of the cells in the image is defined as "damage
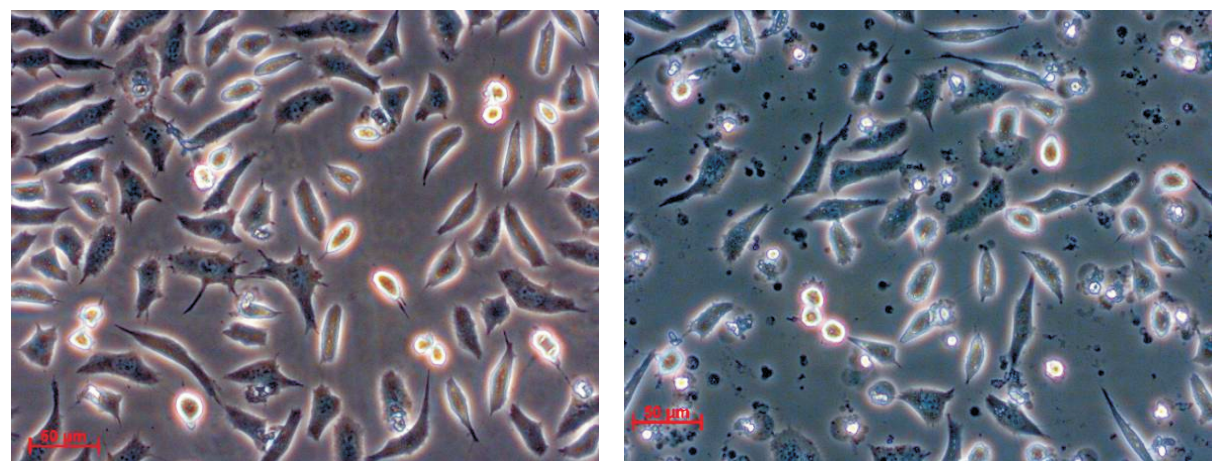

Fig. 2. Original images of an intact cell population (left) and a damaged cell population (right). The calculated confluence: 0.6056 (left), 0.4836 (right). The calculated damage factor: 0.3963 (left), 0.789 (right). 
index" . The damage index was calculated at different time points during cell culture monitoring and for different phenol concentrations (Fig. 4).

\section{Results}

Fifty images were used to develop the CNL rule set; this rule set was then applied to an automatic analysis of 200 test images. In the first analysis, the cell region was separated from the background and the quotient of the cell region to the whole image area was calculated; this is a measurement of the confluence of the cell population in an image (Fig. 1, Fig. 2). In the next step, sub cellular structures such as vacuoles, halos and protrusions were segmented and classified in each of the cells (Fig. 3). The damage factor was automatically calculated for each cell and individual cells were classified as damaged (red), partially damaged (magenta) or healthy (green or yellow). An overall damage factor for each of the images was calculated (Fig. 2, Fig. 3) and the damage index for varying phenol concentrations at different time points during cell culture monitoring was also determined. Fig. 4 shows the results for the damage index plotted as a graph (in blue) and the corresponding results from the cytotoxicity assays.
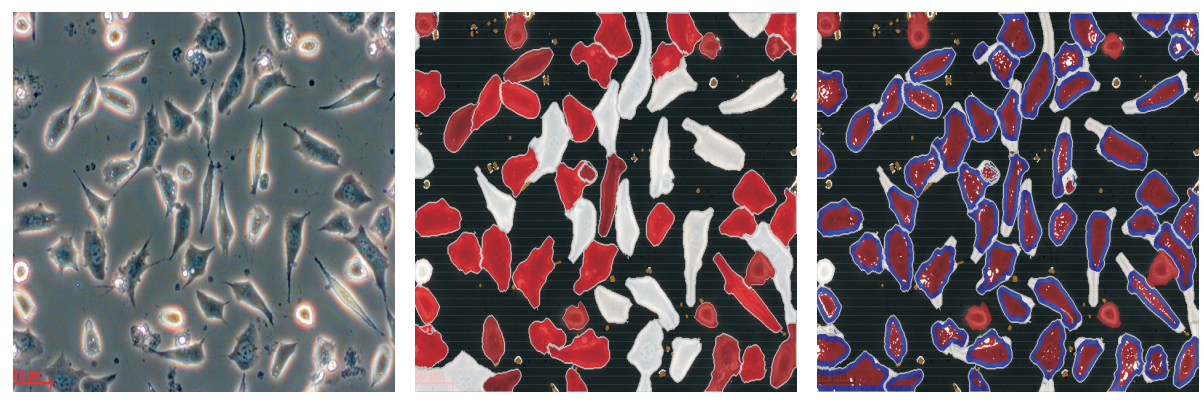

Fig. 3. (a) Original image. (b) Cell separation and classification based on (c) morphology and existence of specific cell contains like vacuoles (bright dots) in the cell body (dark cell region), cell protrusions (gray), halos (bright lines) around the cell body. Because of such cell substructures the cells in b) are classified as intact (bright cells) and damaged (dark cells).

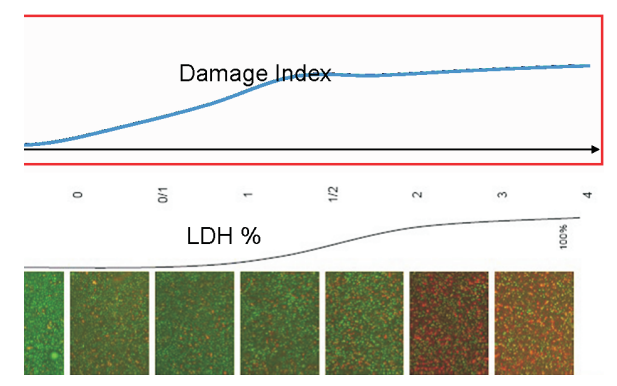

Fig. 4. Damage index. Top: calculated using CNT/CNL image analysis; bottom: LDH \% over the degree of cell damage for the Live/Death assay (fluorescence images). The images show low-damaged (left) and high-damaged cells (right). 


\section{Discussion}

The above work shows that CNL image analysis reproduces the results of the established cytotoxicity assays. Our method can therefore be used to analyze the morphology of single cells in phase contrast images and to quantify the degree of toxic damage of individual cells and the corresponding cell cultures during cell monitoring, as an alternative to semi-automated or manual methods.

Acknowledgement. The authors are grateful to State of Bavaria and Landesgewerbeanstalt Bayern (LGA) for their financial support and Paul Brookes for his help for the manuscript.

\section{References}

1. Debeir O, Adanja I, Warzee N, et al. Phase contrast image segmentation by weak watershed transform assembly. Proc IEEE ISBI. 2008; p. 724-7.

2. Baatz M, Schape A. Multiresolution segmentation an optimization approach for high quality multi-scale image segmentation. In: Angewandte Geographische Informations. Verarbeitung XII. Wichmann; 2000. p. 12-23.

3. Athelogou M, Schönmeyer R, Schmidt G, et al. Bildanalyse in Medizin und Biologie Beispiele und Anwendungen, Medizintechnik. In: Life Science Engineering. Springer; 2008. p. 983-1005. 